\title{
Direct imaging of antigen-antibody binding by atomic force microscopy
}

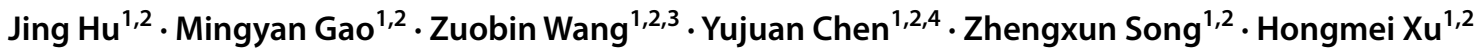

Received: 30 August 2020 / Accepted: 15 September 2020 / Published online: 24 September 2020

(c) King Abdulaziz City for Science and Technology 2020

\begin{abstract}
Direct observation of antigen-antibody binding at the nanoscale has always been a considerable challenging problem, and researchers have made tremendous efforts on it. In this study, the morphology of biotinylated antibody-specific Immunoglobulin E (IgE) immune complexes has been successfully imaged by atomic force microscopy (AFM) in the tapping-mode. The AFM images indicated that the individual immune complex was composed of an IgE and a biotinylated antibody. Excitingly, it is the first time that we have actually seen the IgE binding to biotinylated antibody. Alternatively, information on the length of IgE, biotinylated antibodies and biotinylated antibody-specific IgE immune complexes were also obtained, respectively. These results indicate the versatility of AFM technology in the identification of antigen-antibody binding. This work not only lays the basis for the direct imaging of the biotinylated antibody-IgE by AFM, but also offers valuable information for studying the targeted therapy and vaccine development in the future.
\end{abstract}

Keywords Antigen-antibody binding $\cdot$ Atomic force microscopy $\cdot$ Biotinylated antibody-specific IgE immune complex · Imaging

\section{Introduction}

Antigen-antibody binding research has particular significance in medical diagnosis, vaccine exploration, immunological study and disease mechanism analysis (Nergiz et al. 2019; Bruno et al. 2019). In recent years, many immunoassay

Electronic supplementary material The online version of this article (https://doi.org/10.1007/s13204-020-01558-w) contains supplementary material, which is available to authorized users.

Zuobin Wang

wangz@cust.edu.cn

Yujuan Chen

chenyujuan@cust.edu.cn

1 Ministry of Education Key Laboratory for Cross-Scale Micro and Nano Manufacturing, Changchun University of Science and Technology, Changchun 130022, China

2 International Research Centre for Nano Handling and Manufacturing of China, Changchun University of Science and Technology, Changchun 130022, China

3 JR3CN and IRAC, University of Bedfordshire, Luton LU1 3JU, UK

4 School of Life Sciences, Changchun University of Science and Technology, Changchun 130022, China methods have been used to detect antigen-antibody binding, which include many additional biochemical reagents (Meyer et al. 2020; Stentzel et al. 2015; Zhuang et al. 2019). The extensive use of biochemical agents not only requires tedious process, but also causes serious environmental pollution. In addition, although the immunological techniques were able to monitor and quantify the signal of antigen-antibody, they require a large number of protein samples and there is no way to detect a single molecule. Furthermore, the fatal drawback of these immunological approaches is that they cannot provide a visual image of antigen-antibody binding. Accordingly, it is urgent to find an uncomplicated method to directly observe and determine the specific interactions between antigen and antibody at the molecular level.

Traditionally, electron microscopy, ultrasound imaging and optical microscopy have been widely known as biomolecular imaging methods (Gopal et al. 2019; Negishi et al. 2013; Maresca et al. 2018). However, their actual use has been suffered from some inherent disadvantages, such as complex sample preparation and demanding operating environment (Jung et al. 2010). In 1986, the greatest invention of the atomic force microscopy (AFM) resoundingly opened the door to nano-imaging and nano-manipulating (Krieg et al. 2018). Atomic force microscope is a multi-functional 
biomolecular research instrument with particularly high spatial resolution, exceptionally low invasiveness, and incomparably elegant simplicity, which has attracted increasing attention in the past decades (Alsteens et al. 2017). Actually, many biomolecules, such as proteins and DNAs, have long been satisfactorily imaged by AFM (Ikai et al. 2018). For instance, Kumar et al. (2016) observed the morphologies of RC-LH1-PufX complexes and intact ATP-synthase by gentle tapping-mode AFM. In addition, Valero et al. (2019) performed AFM to image the interlocked double-stranded DNA nanostructures, including rotaxanes, catenanes and daisychain rotaxanes. Additionally, the interaction between proteins and DNA via AFM imaging has also been extensively studied by taking advantage of their morphological differences. Nevertheless, most studies on antigen-antibody interactions have focused on the AFM-based single-molecule force spectroscopy, and only a few are based on AFM imaging (Tanja et al. 2018). Even AFM imaging is studied at its height, and there is very little research on their appearance (Wang et al. 2009; Ouerghi et al. 2002). To the best of our knowledge, the biotinylated antibody-specific IgE immune complexes imaged by AFM have never been reported. While it is conceptually straightforward to use images to analyze the IgE interactions with biotinylated antibodies, the data analysis is complicated by the many conformations of the protein.

In this work, we characterized the interaction between the IgE and biotinylated antibodies by measuring changes in the morphology and length of each sample by tappingmode AFM. It is exciting that AFM image can be used to accurately describe IgE binding to biotinylated antibodies. Moreover, the length data of different samples can enhance the accuracy of our results.

\section{Experimental}

\section{Materials}

IgE and biotinylated antibodies were purchased from human IgE enzyme-linked immunosorbent assay (ELISA) kit (Solarbio Science \& Technology Ltd., Beijing, China). Magnesium chloride hexahydrate $(\mathrm{MgCl} 2 \cdot 6 \mathrm{H} 2 \mathrm{O})$ was obtained from Beijing Chemical Works Ltd. (Beijing, China). The $10 \mathrm{~mm} \times 10 \mathrm{~mm}$ fluorphlogopite mica was supplied by Taiyuan Fluorphlogopite Mica Ltd. (Changchun, China). All the chemicals were used as received without purification during the study. Ultrapure water with resistivity no less than 18.2 M $\Omega \cdot \mathrm{cm}$ was employed in the experiments.

\section{Detection of IgE}

Experimentally, IgE was determined by an ELISA kit in accordance with the manufacturer's specifications.

\section{Atomic force microscopy imaging}

To image the antibody-specific IgE immune complexes, the sample was prepared as displayed in Fig. 1. In a typical experiment, $20 \mu \mathrm{L}$ of aqueous $\mathrm{MgCl}_{2} \cdot 6 \mathrm{H}_{2} \mathrm{O}(50 \mathrm{mM})$ solution was first incubated on the surface of newly cleaved mica for $1 \mathrm{~min}$, and then $10 \mu \mathrm{L}$ of $\operatorname{IgE}\left(1 \mathrm{ng} \mathrm{mL}{ }^{-1}\right)$ solution was dripped. After $10 \mathrm{~min}$, the surface covering the IgE was exposed to a biotinylated antibody solution (10 $\mu \mathrm{L}, 1 \mathrm{ng} \mathrm{mL}{ }^{-1}$ ) for $60 \mathrm{~min}$. Finally, the mica surface was thoroughly rinsed with water for three times and dried with nitrogen. Similarly, the $\mathrm{Mg}^{2+}$-modified mica surfaces covered with the IgE and biotinylated antibodies were also obtained, respectively. For comparison, the sample without the protein solution was prepared according to the above method.

The surface morphology measurement and data acquisition of all samples were carried out by the AFM system (Nano Wizard III, Germany) with the tapping-mode in air. The Tap300AI-G cantilevers (resonance frequency: $300 \mathrm{kHz}$, spring constants: $40 \mathrm{~N} \mathrm{~m}^{-1}$, curvature radius: $10 \mathrm{~nm}$ ) were used for the experiment, and the $256 \times 256$ pixels images were collected at the line rate of $1 \mathrm{~Hz}$.

\section{Results}

After unremitting efforts, a Japanese couple Teruko and Kimishige Ishikaka discovered the skin-sensitizing IgE in 1967 (Ribatti 2016). The discovery of IgE was a major breakthrough in the area of immunology and allergy (Hirohisa et al. 2019). Since IgE plays an absolutely essential role in allergic responses and can be used for the diagnosis and treatment of allergic diseases (Han et al. 2020), it is crucial to understand the interaction between the specific antigens and $\operatorname{IgE}$ antibodies.

Herein, the binding of IgE to biotinylated antibodies was first examined by the classic ELISA method, and the corresponding results were obtained, as shown in Fig. 2. It can be seen that the absorbance tends to grow in proportion to the IgE concentration, which is in fair agreement with the manufacturer's instructions, indicating a good affinity between the IgE and biotinylated antibodies. Nevertheless, the minimum detection limit of ELISA method was $0.24 \mathrm{ng} \mathrm{mL}^{-1}$, which could not meet our requirement 


\section{Mica}

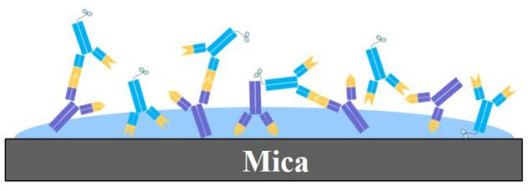

$\mathrm{MgCl}_{2} \cdot 6 \mathrm{H}_{2} \mathrm{O}$ solution

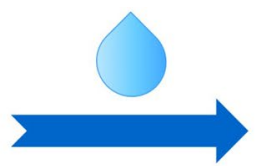

Mica
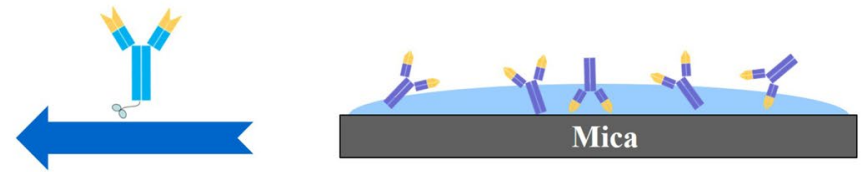

IgE

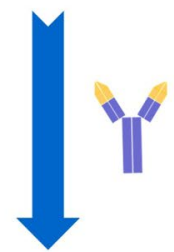

Mica

Fig. 1 Schematic drawing for the preparation of antibody-specific IgE immune complexes

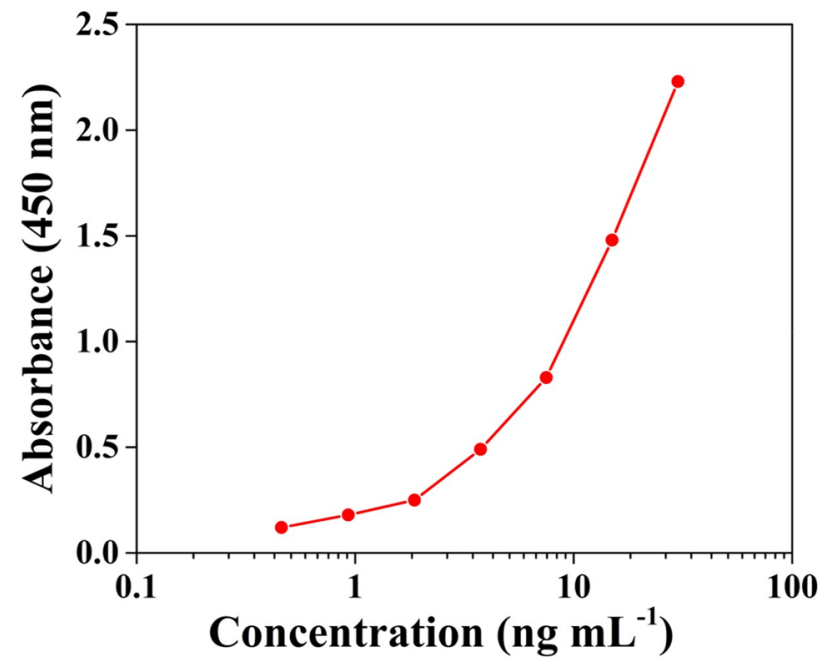

Fig. 2 ELISA results for the measurement of biotinylated antibodyIgE immune complexes

to study the interaction between antigen and antibody at the molecular level.

To gain an insight into the details of a single IgE binding to a single biotinylated antibody, the AFM was further employed to visualize the morphology of $\mathrm{IgE}$ before and after incubation with biotinylated antibodies, and the relevant data were also obtained. There was no significant difference in the AFM image morphology of mica surface before and after the modification of $\mathrm{Mg}^{2+}$ (Fig. S1). Therefore, the modification of $\mathrm{Mg}^{2+}$ had no effect on the observation of mica surface proteins at the current resolution scale. Additionally, the mica surface modified by $\mathrm{Mg}^{2+}$ was very smooth, while some randomly distributed nanoparticles were found on the mica surface after incubation with $\operatorname{IgE}$ and biotinylated antibodies (Fig. 3), indicating that these proteins had been successfully adsorbed on the $\mathrm{Mg}^{2+}$-modified mica surface, respectively. As shown in Fig. 3a and b, the IgE molecules in various orientations individually dispersed on the surface of mica, which was consistent with our previous results (Hu et al. 2019). An analogous phenomenon was observed in Fig. 3c and d, where the biotinylated antibodies with different conformations were found to be evenly adsorbed on the mica surface. Interestingly, there was a lot of little tail-like structures appeared in the view. It must be kept in mind that the biotinylated antibodies used in this study are biotinylated anti-human IgE antibodies, which are immunoglobulin $\mathrm{G}(\mathrm{IgG})$ antibodies with biotins. Furthermore, IgG shares the similar molecular architecture as IgE, consisting of two antigen binding fragments (Fab) and one crystallizable fragment (Fc), and appears as a single globular morphology in most cases (Funari et al 2016; Gould and Sutton 2008). Hence, it would be reasonable to believe that the biotin attached to IgG molecule possesses the little taillike structure. However, it can be seen from the figures that not every IgG is connected to a biotin. This phenomenon can be attributed to the following two principal reasons. First, 
Fig. 3 AFM height (a and c) and three-dimensional (b and d) images of the $\operatorname{IgE}$ (a and $\mathbf{b}$ ) and biotinylated antibodies (c and $\mathbf{d})$ (a)

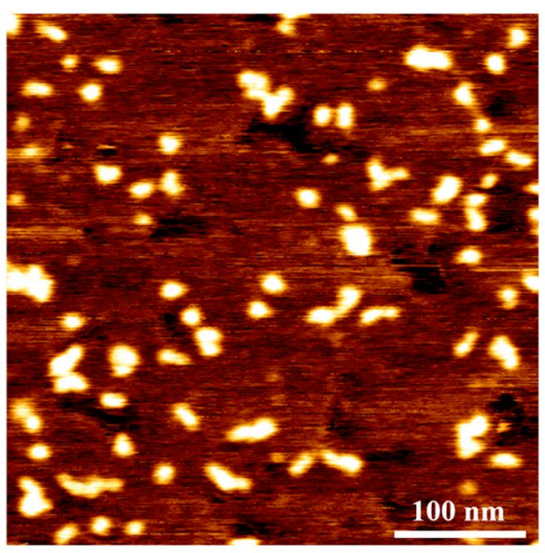

(c)

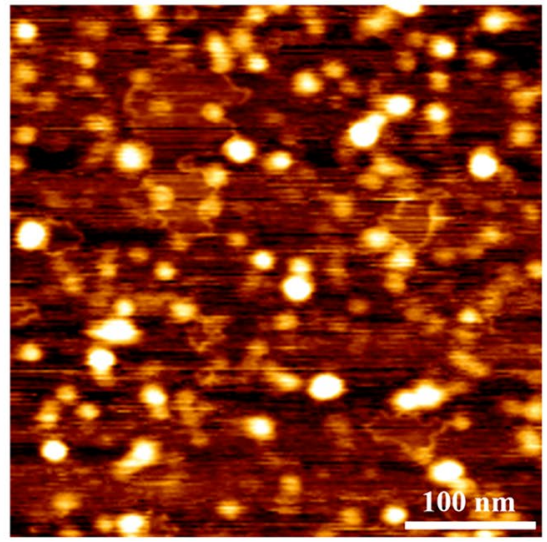

(b)
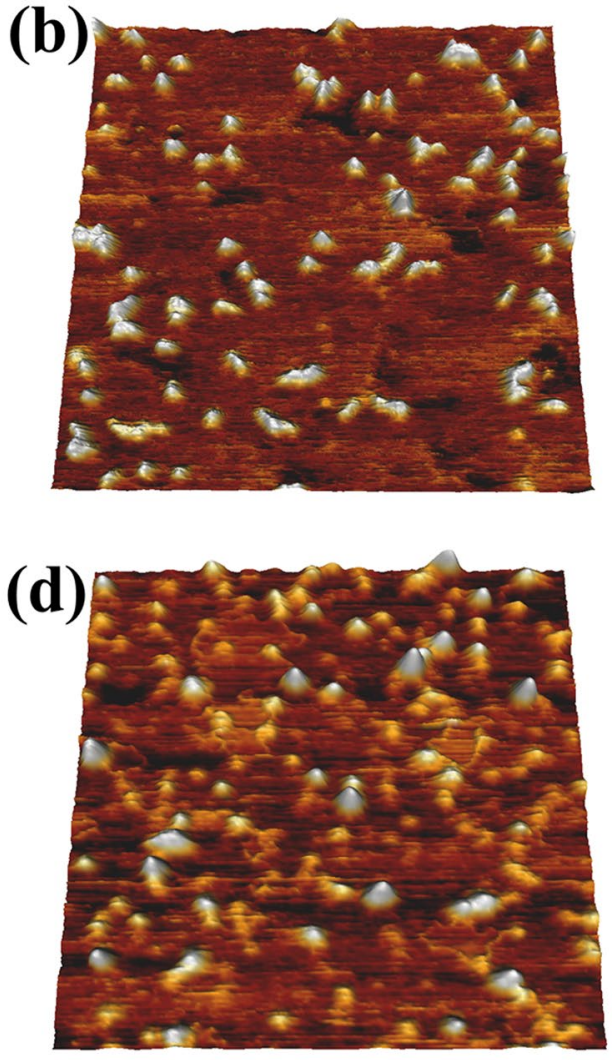

Table 1 The length distribution of IgE and biotinylated antibodies
Fig. 4 AFM phase (a) and height (b) images of the $\mathrm{IgE}$ after incubation with biotinylated antibodies

\begin{tabular}{lll}
\hline Length & \multicolumn{2}{l}{ Frequency } \\
\cline { 2 - 3 } & IgE & $\begin{array}{l}\text { Bioti- } \\
\text { nylated } \\
\text { antibodies }\end{array}$ \\
\hline $13-16 \mathrm{~nm}$ & $2 \%$ & $4 \%$ \\
$16-19 \mathrm{~nm}$ & $16 \%$ & $12 \%$ \\
$19-22 \mathrm{~nm}$ & $24 \%$ & $28 \%$ \\
$22-25 \mathrm{~nm}$ & $30 \%$ & $32 \%$ \\
$25-28 \mathrm{~nm}$ & $20 \%$ & $14 \%$ \\
$33-34 \mathrm{~nm}$ & $8 \%$ & $10 \%$ \\
\hline
\end{tabular}

the biotinylated antibodies were randomly distributed on the mica surface. Second, their structures were imaged from the top view, and various morphologies could be obtained. Besides that, the length of $\operatorname{IgE}$ and biotinylated antibodies were also calculated from more than 50 molecules, respectively. As shown in Table 1, the length of IgE was mainly between 22 and $25 \mathrm{~nm}(30 \%)$, which is close to the length of the biotinylated antibodies ( $22 \mathrm{~nm}$ and $25 \mathrm{~nm}, 32 \%$ ). It is worth mentioning that the statistical length of the biotinylated antibodies does not include the biotin length. (a)

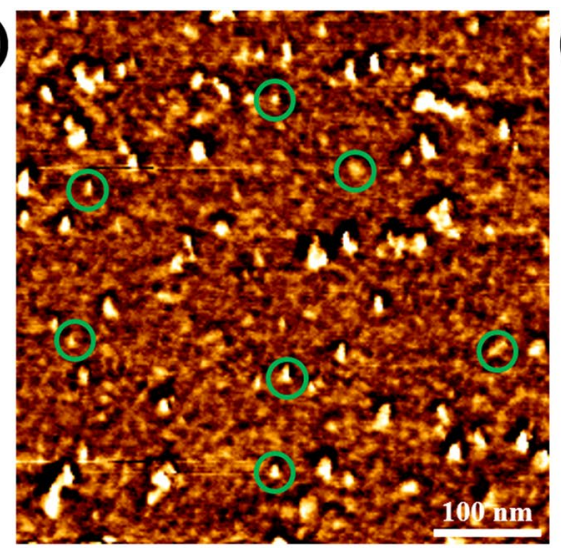

(b)

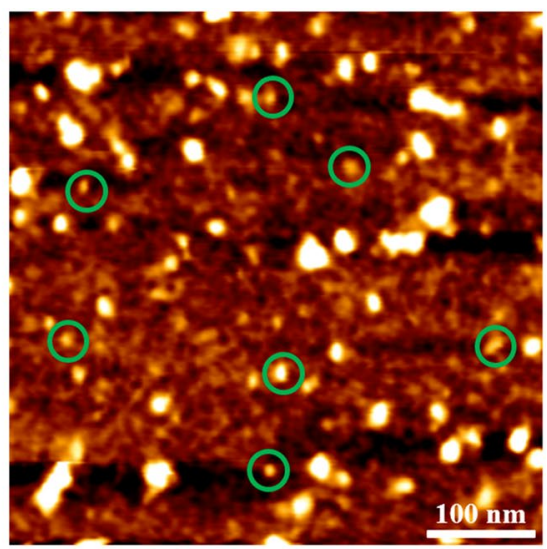


It should be noted that after incubation with biotinylated antibodies, the IgE morphology changed significantly, resulting in a new characteristic image that was completely different from either IgE or biotinylated antibody alone (Fig. 4). Furthermore, compared with the single IgE and biotinylated antibody molecules, some larger particles (Fig. 5) could be detected in the field of view. It is worth noting that the corresponding results are derived from calculations and statistics of more than 50 particles. These results strongly demonstrate that the IgE has reacted with biotinylated antibodies, and we have successfully probed the biotinylated antibody-specific IgE immune complexes. Although it is easy to track and image individual biotinylated antibody-specific IgE immune complexes that are well isolated from particles, not all of these complexes can be tracked, even on well-dispersed images. The untracked complexes included those that clustered with other particles, as well as those that were not completely within the image boundaries. In addition to the immune complexes, a number of nanoparticles were also observed with the structures corresponded to the IgE, biotinylated antibodies and their detached fragments (The circles in Fig. 4 are typical representations of the structures).

As observed, only some of the complexes were detected in the Fig. 4, and some of the $\operatorname{IgE}$ and biotinylated antibodies were not involved in the formation of the antigen-antibody complex. The main reason of this phenomenon is that the binding of $\operatorname{IgE}$ to the biotinylated antibodies depends on the ability of the antibodies to capture biotinylated antibodies, and this specific ability is largely dependent on the orientation and conformation of the antibody. In the case of physical adsorption, when the IgE orientation is random, only a small fraction of the adsorbed antibodies can capture biotinylated

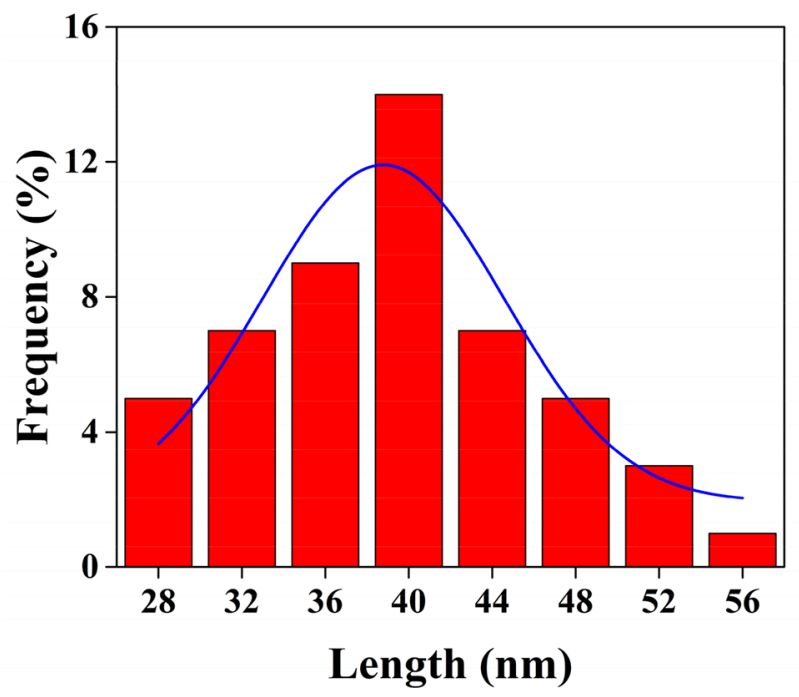

Fig. 5 The length distribution histogram of the biotinylated antibodyspecific IgE immune complexes antibodies and retain them. In fact, the biotinylated antibodyspecific IgE immune complexes have a wide variety of morphologies and sizes on the mica surface due to their threedimensional structures. It was extremely difficult to find all the orientations of the biotinylated antibody-specific $\operatorname{IgE}$ immune complexes. Hence, we randomly selected several immune complex molecules with high resolution to reveal interesting details about them, and their corresponding AFM phase, three-dimensional and possible spatial images were shown in Fig. 6a-d, e-h and i-l, respectively. Specifically, Fig. 6a, e and i would correspond to the biotinylated antibody-specific IgE immune complex deposited on the surface by the $\mathrm{Fab}$ and $\mathrm{Fc}$ fragments of $\mathrm{IgE}$ or biotinylated antibody. Furthermore, when the immune complex is attached to the support via the IgE Fab fragment and the three fragments of biotinylated antibody, it would be shown in Fig. $6 \mathrm{~b}, \mathrm{f}$ and $\mathrm{j}$. Additionally, the immune complex molecule could also flat on the mica surface by its six fragments. The immune complex with this conformation would produce the images as shown in Fig. 6c, g and k. Meanwhile, Fig. 6d, h and 1 would represent the biotinylated antibody-specific IgE immune complex adsorbed on the mica surface by the IgE fragment and the two Fab fragments of biotinylated antibody. It is also possible that the immune complex is located on the support by the IgE Fab and $\mathrm{Fc}$ fragments and the two Fab fragments of biotinylated antibody. Overall, the quite good match of the 3D complex revealed the molecular mechanism by which a single biotinylated antibody interacts with one of the two Fab fragments immobilized on the mica surface with IgE. From Fig. 6a-d, we can find that the biotin is sometimes linear and sometimes blocky, and the linear structure is easier to be discriminated than the blocky structure. There is no doubt that the flexible three-dimensional structure results in different forms of biotin on the mica surface. Moreover, the tip moves over the tight biotin making the biotin image blurry.

\section{Discussion}

In modern medicine, antibody-targeted immunotherapy is a powerful tool for the treatment of cancer and autoimmune diseases. The growing prevalence of antibody-targeted treatment requires a deeper understanding of the functions of antibodies, especially their interaction with antigens ( $\mathrm{Lu}$ and Sun 2015). Affinity is not only a vital parameter to determine the interaction between molecules, but also an important indicator to understand the molecular recognition, biological process, drug discovery and screening (Oh et al. 2019; Linke et al. 2015). The results from the ELISA depicted that the IgE had a high affinity with biotinylated antibodies, which laid a foundation for further observation of biotinylated antibody-specific IgE immune complexes. 
(a)

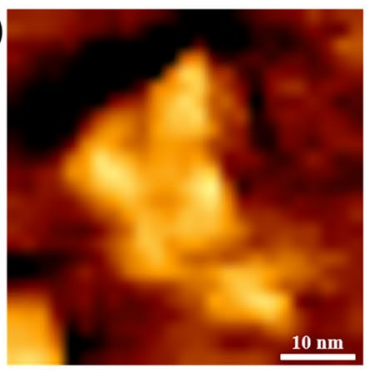

(b)

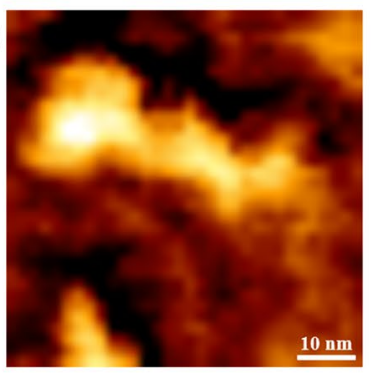

(c)

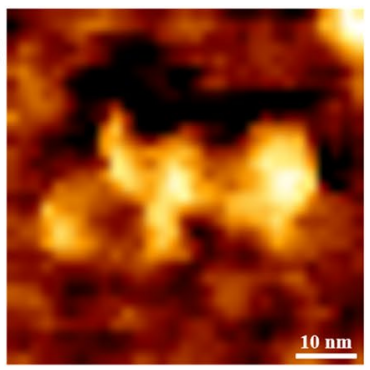

(d)
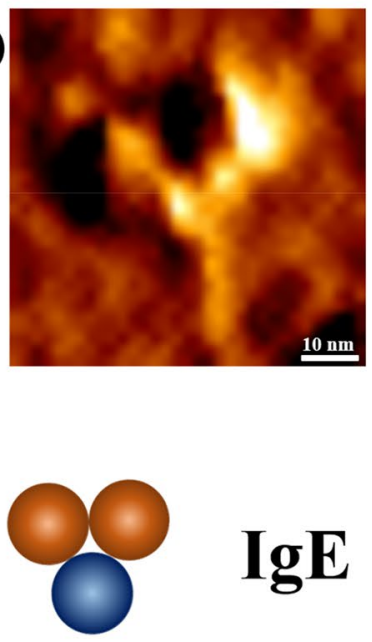

(e)

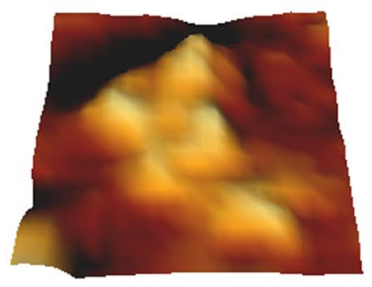

(f)

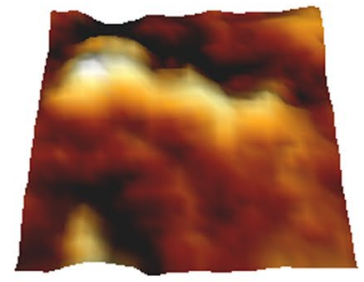

(g)

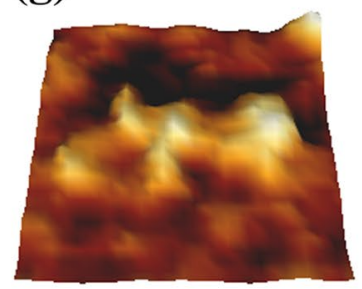

(h)

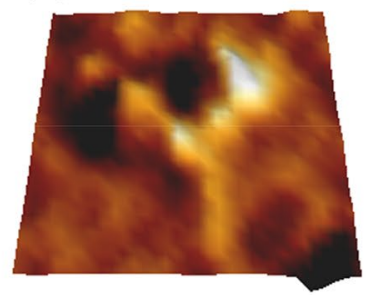

(i)

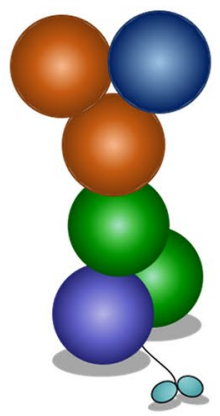

(j)

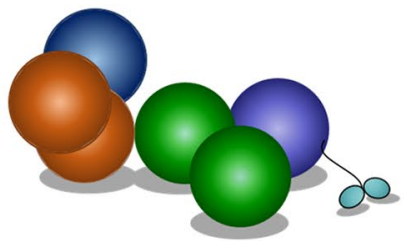

(k)

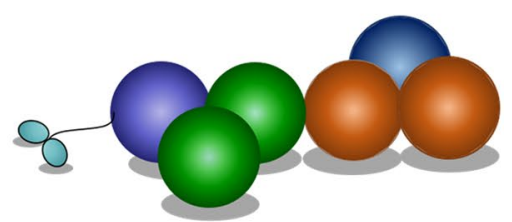

(l)

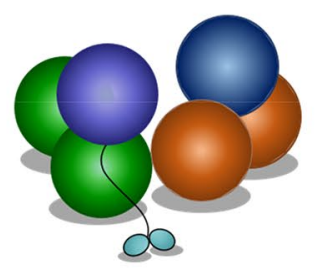

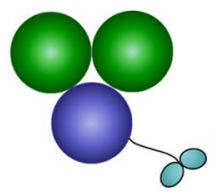

\section{Biotinylated antibody}

Fig. 6 AFM phase (a, b, $\mathbf{c}$ and $\mathbf{d})$, three-dimensional $(\mathbf{e}, \mathbf{f}, \mathbf{g}$ and $\mathbf{h})$ and spatial $(\mathbf{i}, \mathbf{j}, \mathbf{k}$ and $\mathbf{l})$ images of the individual biotinylated antibodyspecific IgE immune complexes

As expected, the results from AFM indicate that the different orientations of the antibody-specific IgE immune complexes are formed on the mica surface, from which the $\operatorname{IgE}$ and biotinylated antibodies can be readily identified because the biotinylated antibodies possess a little tail-like structure. Admittedly, there are some immune complexes that confuse
$\operatorname{IgE}$ and biotinylated antibodies due to the inevitable factors of the tightness of the immune complexes themselves and the randomness of their distribution on the mica surface. In addition, due to the highly variable in the length of the antibody-specific IgE immune complexes, we made a statistical analysis of the relevant data. Although the length of these antibody-specific 
IgE immune complexes vary slightly in the range of $28-56 \mathrm{~nm}$, it is consistent with the expected length of single immune complex. Moreover, since the length of the immune complex is larger than that of $\mathrm{IgE}$ and biotinylated antibody, we can also determine whether the $\operatorname{IgE}$ is bound to the biotinylated antibodies by the particle length. Thus, in our case, it is possible to distinguish between the antigen, antibody and antigen-antibody immune complex by the length and topography.

\section{Conclusion}

Obtaining knowledge about the topological structure of the proteins and their interactions is the key to understanding the fundamental causes of disease and developing drugs. In this respect, AFM imaging technology provides a unique possibility for the study of proteins. In this paper, the morphology and length of IgE, biotinylated antibodies and biotinylated antibody-specific IgE immune complexes were analyzed by AFM, respectively. The results of AFM imaging demonstrated that the immune complexes exhibited various morphologies, and we were able to identify the IgE and biotinylated antibody based on the protein morphology in most cases. Moreover, the individual immune complex shows the larger size in length compared with the $\mathrm{IgE}$ and biotinylated antibody. By combining the above information, we can quickly determine whether IgE is bound to biotinylated antibodies. Therefore, the morphology and length analysis can be extremely helpful to investigate antigen-antibody interactions at the molecular level and provide a new way for the studies of vaccines and targeted therapies. We are confident that our successful visualization of the biotinylated antibody-specific IgE immune complexes at the single molecular scale will also enable the direct imaging of other immune complexes.

Acknowledgements We are grateful to the National Key R \& D Program of China (No. 2017YFE0112100), EU H2020 Program (MNR4SCELL No. 734174), Jilin Provincial Science and Technology Program (Nos. 20160623002TC, 20180414002GH, 20180414081GH, 20180520203JH, 20190702002GH, 20190201287JC and 20200901011SF), and "111" Project of China (D17017). This work was also partially supported by Changli Nano Biotechnology (China).

\section{Compliance with ethical standards}

Conflict of interest The authors declare no conflict of competing interest.

\section{References}

Alsteens D, Gaub HE, Newton R et al (2017) Atomic force microscopy-based characterization and design of biointerfaces. Nat Rev Mater 2(5): 17008

Bruno V, Ana M, Tomás RC et al (2019) Antibody modified gold nanoparticles for fast colorimetric screening of rheumatoid arthritis. Analyst 144:3613-3619
Funari R, Della VB, Altucci C et al (2016) Single molecule characterization of UV-activated antibodies on gold by atomic force microscopy. Langmuir 32(32):8084-8091

Gould HJ, Sutton BJ (2008) IgE in allergy and asthma today. Nat Rev Immunol 8(3):205-217

Gopal S, Chiappini C, Armstrong JPK (2019) Immunogold FIBSEM: combining volumetric ultrastructure visualization with 3D biomolecular analysis to dissect cell-environment interactions. Adv Mater 31(32): 1900488

Han XS, Cao MD, Zhou BC et al (2020) Specifically immobilizing His-tagged allergens to magnetic nanoparticles for fast and quantitative detection of allergen-specific IgE in serum samples. Talanta 219:121301

Hirohisa S, Harald R (2019) Landmark papers in our journal: Articles I to III of the series describing the discovery of IgE by the Ishizakas. J Allergy Clin Immun 144(5):1163-1165

Hu J, Gao MY, Wang Y et al (2019) Imaging the substructures of individual $\mathrm{IgE}$ antibodies with atomic force microscopy. Langmuir 35(46): 14896-14901

Ikai A, Afrin R, Saito M et al (2018) Watanabe-Nakayama, T. Atomic force microscope as a nano- and micrometer scale biological manipulator: a short review. Semin Cell Dev Biol 73:132-144

Jung SH, Park D, Park JH et al (2010) Molecular imaging of membrane proteins and microfilaments using atomic force microscopy. Exp Mol Med 42(9):597-605

Krieg M, Fläschner G, Alsteens D et al (2018) Atomic force microscopy-based mechanobiology. Nat Rev Phys 1(1):41-57

Kumar S, Cartron ML, Mullin N et al (2016) Direct imaging of protein organization in an intact bacterial organelle using highresolution atomic force microscopy. ACS Nano 11(1):126-133

Linke A, Jungbauer SH, Huber SM et al (2015) Potent affinity material for tracing acetone and related analytes based on molecular recognition by halogen bonds. Chem Commun 51(11):2040-2043

Lu J, Sun PD (2015) Structural mechanism of high affinity Fc $\gamma$ RI recognition of immunoglobulin G. Immunol Rev 268(1):192-200

Maresca D, Sawyer DP, Renaud G et al (2018) Nonlinear X-wave ultrasound imaging of acoustic biomolecules. Phys Rev X $8(4): 041002$

Meyer TC, Schmidt F, Steinke J et al (2020) Technical report: xMAPr-High-Dynamic-Range (HDR) quantification of antigenspecific antibody binding. J Proteom 212:103577

Negishi Y, Hamano N, Tsunoda Y et al (2013) AG73-modified bubble liposomes for targeted ultrasound imaging of tumor neovasculature. Biomaterials 34(2):501-507

Nergiz Y, Elif BA, Mustafa KS (2019) An epoxysilane modified indium tin oxide electrode for the determination of PAK 2: application in human serum samples. Anal Chim Acta 11(35):31729-31734

Oh YJ, Koehler M, Lee Y et al (2019) Ultra-sensitive and label-free probing of binding affinity using recognition imaging. Nano Lett 19(1):612-617

Ouerghi O, Touhami A, Othmane A et al (2002) Investigating antibody-antigen binding with atomic force microscopy. Sens Actuators B 84(2):167-175

Ribatti D (2016) The discovery of immunoglobulin E. Immunol Lett 171:1-4

Stentzel S, Sundaramoorthy N, Michalik S et al (2015) Specific serum IgG at diagnosis of staphylococcus aureus bloodstream invasion is correlated with disease progression. J Proteom 128:1-7

Tanja DB, Stefan N, Raimund G et al (2018) Single molecule force spectroscopy reveals two-domain binding mode of pilus-1 tip protein RrgA of streptococcus pneumoniae to fibronectin. ACS Nano 12:549-558 
Valero J, Centola M, Ma Y et al (2019) Design, assembly, characterization, and operation of double-stranded interlocked DNA nanostructures. Nat Protoc 14(10):2818-2855

Wang MS, Zameer A, Emadi S et al (2009) Characterizing antibody specificity to different protein morphologies by AFM. Langmuir 25:912-918
Zhuang QQ, Deng HH, He SB et al (2019) Immunoglobulin G-encapsulated gold nanoclusters as fluorescent tags for dot-blot immunoassays. ACS Appl Mater Inter 11(35):31729-31734 